\title{
A COMPARATIVE STUDY FOR WHEELED MOBILE ROBOT PATH PLANNING BASED ON MODIFIED INTELLIGENT ALGORITHMS
}

\author{
Muna M. AL -Nayar \\ Muna_alnayar@yahoo.com \\ Control and Systems Eng. \\ University of Technology
}

\author{
Khulood E. Dagher \\ dagerkhulood@yahoo.com \\ AL- Kawarizmi Engineering college \\ University of Baghdad
}

\author{
Esraa A. Hadi \\ engesraa753@yahoo.com \\ Control and Systems Eng. \\ University of Technology
}

\begin{abstract}
From the time being, there are even instances for application of mobile robots in our life like in home, schools, hospitals, etc. The goal of this paper is to plan a path and minimizing the path lengths with obstacles avoidance for a mobile robot in static environment. In this work we depict the issue of off-line wheeled mobile robot (WMR) path planning, which best route for wheeled mobile robot from a start point to a target at a plane environment represented by 2-D work space. A modified optimization technique to solve the problem of path planning problem using particle swarm optimization (PSO) method is given. PSO is a swarm intelligence based stochastic optimization technique which imitate the social behavior of fish schooling or bird flocking, was applied to locate the optimum route for mobile robot so as to reach a target. Simulation results, which executed using MATLAB 2014 programming language, confirmed that the suggested algorithm outperforms the standard version of PSO algorithm with the same environment conditions by providing the shortest path for mobile robot.
\end{abstract}

KEYWORDS: Wheeled Mobile Robot, Path planning, Static Environment, and Particle Swarm Optimization Algorithm, Chaotic PSO.

\section{دراسة مقارنة لتخطيط مسار الروبوت المتحرك بعجلات استتادا الى خوارزميات ذكية معدلة

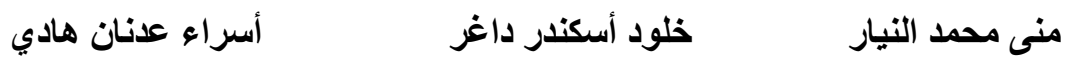

في الوقت الحالي هنالك عدد من التطبيقات للروبوتات المتنقلة في حياتنا مثل المنزل و المدارس و المستشفيات، الخ. الهدف

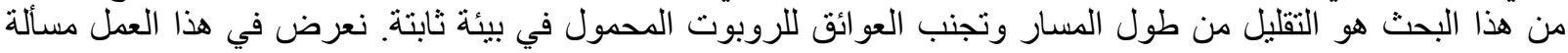

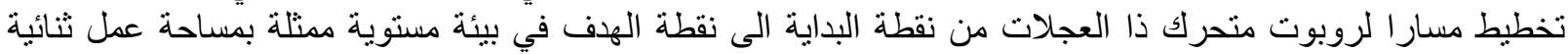

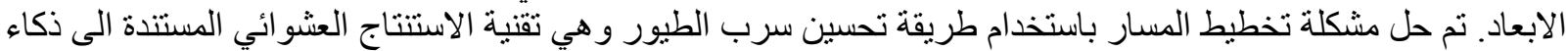

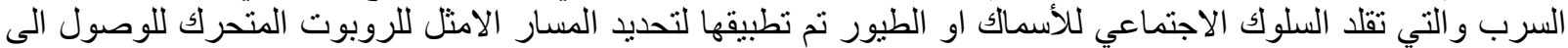

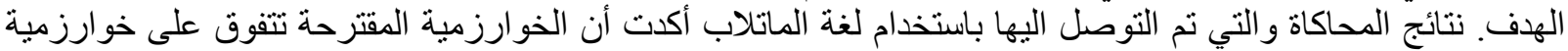

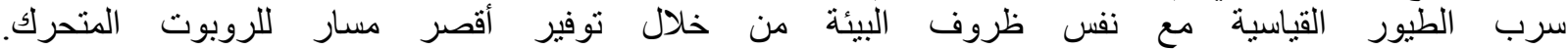
الكلمات المفتاحية: الروبوت المتحرك بعجلات، تخطيط المسار، البيئة الثابتة، خوارزمية سرب الطيور، خوارزمية سرب الطيور المشوشة . 


\section{INTRODUCTION}

In general, a large number of studies and articles count the wheeled mobile robots as a major subject of large benefit due to their evolutionary applications Al-Araji [ 2014]. Mobile robots have been successfully applied in many fields such as medical and military applications, space exploration, public and domestic duties. They can implement difficult and hazardous jobs with complex requirements and often have to do so autonomously, without the help of a human operator Cholodowicz, et.al,[2017]. Path planning is a significant issue in navigation of mobile robots. The goal is to detect a best and collision -free path from a source point to a destination point in a predefined work space. In any event, there are a number of routes for mobile robot to accomplish the goal, but actuality, the desired route is picked according to several optimization criteria. These optimization criteria are: shortest route length, least energy consuming or minimum time with the shortest route length is the most adopted criteria [Abbas, et.al, 2016]. The mobile robot path planning issue is commonly work out as describes: specified a wheeled mobile robot and definition of a work space, plan a route between two fixed positions, a source and goal point [Mnubi, 2016]. The path planning environment can be classified into two main types : static and dynamic. In the static environment, the entire routes should be form before beginning pursuance. While, for dynamic environment, re-planning are wanted from time to time and additional update time is required .Respecting on the environment kind, path planning approaches are divided into two classes, which are online and offline techniques .They might also divided into conventional and soft computing techniques. Conventional path planning methods such as Artificial Potential Field (APF), Road map and Cell decomposition approaches. As to soft computing methods, they include Particle Swarm Optimization (PSO), Neural Networks (NNs), Ant Colony Optimization (ACO), Fuzzy Logic (FL) and Genetic Algorithms (GAs). Clearly, each algorithm has its own power points and flaw points which motivate investigators to treat options and more effective algorithms [Jalel, et.al, 2015]. The rest of this paper is ordered as follows: section 2 describes the related work; section 3 describes a model of wheeled mobile robot; section 4 describes the path planning problem; section 5 describes the classic PSO algorithm; section 6 describes the Chaotic PSO algorithm and simulation results and discussion are presented in section 7.Finally, section 8 gives the research conclusions.

\section{RELATED WORKS}

There are many kinds of research studies path planning in recent years. In 2014, N. H. Abbas et al, describes the use of directed Artificial Bee Colony algorithm to get the best route from start position to goal position in free-space environment. That research offers that the proposed algorithm is efficient and acquire routes with pleasurable products [Abbas, et.al, 2014]. In 2015, S. Jalel et al, introduced a new mobile robot path planning technique by using NURBS (Non Uniform Rational B-spline) curve designing using GA to perform the optimal route between start and goal points [Jalel, et.al, 2015]. In 2016, T. A. Jaleel et al, suggested a hybrid control methodology using improved APF with modified CSA to path planning of decentralized method for multi mobile robot in moving environment. Simulation results offer that the suggested technique lack to locate an entire, optimum and collision -free path for multi mobile robot [Jaleel, et.al, 2016]. In 2015 A. A. Ahmed et al, performed path planning in unknown environment by combining APF approach with Fuzzy Logic Control (FLC) to think through disadvantages of Artificial Potential Field (APF) approach like local minima issues, create an efficient path planning and develop the goodness of the mobile robot path planning [Ahmed et.al, 2015]. Various algorithms have been proposed for this purpose, Particle Swarm Optimization (PSO) is one of the most used algorithms. 


\section{WHEELED MOBILE ROBOT PARADIGM}

In general, mobile robot is a type of electromechanical system [Al-Araji, 2014]. The paradigm as shown in Fig. 1 consists of a cart with two driving wheels mounted on the same axis and an omni-directional castor in the face of cart. The castor carries the mechanical body and saves the platform more stable. The radius of left and right wheel is $r$, the distance between the two wheels is L, while the center of wheeled mobile robot mass is c.

The position and orientation vector in the global coordinate $[\mathrm{O}, \mathrm{X}, \mathrm{Y}]$ of the mobile robot is located as equation (1):

$$
q=(x, y, \theta)^{T}
$$

Where the real position of the mobile robot is denoted as ( $\mathrm{x}$ and $\mathrm{y}$ ) while the orientation of the mobile robot is denoted as $(\theta)$. The coordinates $(\mathrm{x}, \mathrm{y}, \theta)$ depict the arrangement of the wheeled mobile robot.

Based on a non-holonomic constraints as in equation (2) [Al-Araji, 2014], the kinematic equations of the mobile robot model can be described in equations $(3,4,5)$ after achievement the two conditions, the first one is a pure rolling wheels and the second is non-slipping wheels.

$$
\begin{aligned}
& -\dot{x}(t) \sin \theta(t)+\dot{y}(t) \cos \theta(t)=0 \\
& \dot{x}(t)=V I(t) \cos (\theta(t)) \\
& \dot{y}(t)=V I(t) \sin (\theta(t)) \\
& \dot{\theta}(t)=V w(t)
\end{aligned}
$$

Where, VI is represented the linear velocity while $\mathrm{Vw}$ is represented the angular velocity.

The reference linear velocity for the optimal path is described in equation (6), while the reference angular velocity is describe in equation (7) [Al-Araji, et.al, 2011]

$$
\begin{aligned}
& V r=\sqrt{(\dot{x} \dot{r})^{2}+(\dot{y} \dot{r})^{2}} \\
& W r=\frac{\ddot{y r} \dot{x} r-\ddot{x} \dot{y} r}{(\dot{x} \dot{r})^{2}+(\dot{y} r)^{2}}
\end{aligned}
$$

Consequently, the right wheel velocity and left wheel velocity can be calculated as in Equation (8) [Al-Araji, et.al, 2011] based on equations (6 and 7).

$$
\left[\begin{array}{l}
V L(t) \\
V R(t)
\end{array}\right]=\left[\begin{array}{c}
v r+\frac{L}{2} w r \\
v r-\frac{L}{2} w r
\end{array}\right]
$$

\section{PATH PLANNING PROBLEM}

The robot path planning issue is very challenging in robotics. The major aim is to find a desired route between start and target points. Robot navigation issue has to be interested in two essential elements: accuracy and safety. That means transact with finding obstacle avoidance route and attached the desired path. [Abdulsahib , 2016]. 


\section{OPTIMIZATION TECHNIQUES}

\section{Standard Particle Swarm Optimization (PSO) Algorithm}

Particle Swarm Optimization is a meta-heuristic search technique. It means algorithm update the information at each iteration. Particle Swarm Optimization was developed in 1995 by James Kennedy and Russell Eberhart which is a Swarm Intelligence (SI) based stochastic optimization technique. Swarm intelligence is emerging research area which is similar to genetic algorithm. Swarm intelligence used to solve optimization and cooperative problems among intelligent particles [Abbas, et.al, 2016].

In the next iteration these particles are update their velocity according to equations $(9,10)$ :

$$
\begin{aligned}
& V i^{x}(k+1)=W V i+c 1 r 1[\text { pbesti }(k)-x i(k)]+c 2 r 2[\text { gbest }(k)-x i(k)] \\
& V i^{y}(k+1)=W V i+c 1 r 1[\text { pbesti }(k)-y i(k)]+c 2 r 2[\text { gbest }(k)-y i(k)] \\
& \quad \mathrm{i}: 1,2,3 \ldots \ldots \mathrm{N}_{\text {pop }} \\
& \mathrm{k}: \text { the current iteration ...... Maxit }
\end{aligned}
$$

Where $\mathrm{N}_{\text {pop }}$ is the size of the swarm, Maxit is the maximum no. of iterations, $\mathrm{r} 1$ and $\mathrm{r} 2$ are the random functions between $[0,1], \mathrm{W}$ is the inertia weight, $\mathrm{c} 1$ is the personal learning coefficient , c2 is the global learning coefficient, pbest is the best weight of each particle and gbest is the best particle among all the particles in the population.

In the next iteration these particles are then move to next position according to equations (11, 12):

$$
\begin{gathered}
X i(k+1)=X i(k)+V i^{x}(k+1) \\
Y i(k+1)=Y i(k)+V i^{y}(k+1)
\end{gathered}
$$

PSO algorithm can be summarized in pseudo code as shown below:

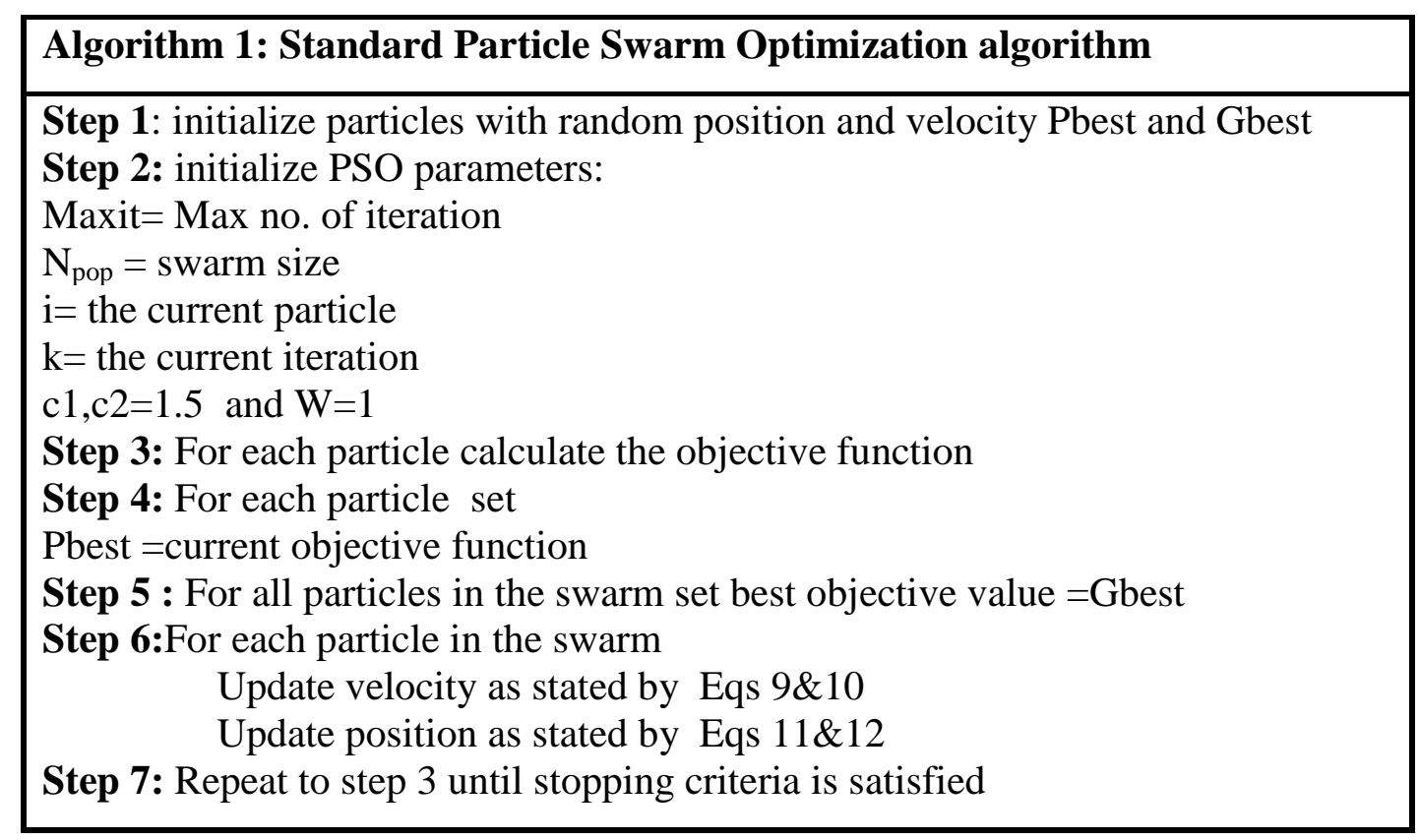




\section{Chaotic Particle Swarm Optimization (CPSO)}

Chaotic PSO is a stochastic search technique that differs from any of the present swarm intelligence approaches and evolutionary computation. Chaotic PSO can execute global searches at higher speeds than stochastic techniques that depend on probabilities [Assarzadeh, et.al, 2016].

The PSO Technique is a new evolution technology, has many power point, such as simple algorithm and quick convergence, but in the last stage of iterations of the algorithm when all particles approach the optimal solution, the convergence may become slow and the solution precision may not be absolutely satisfactory. The chaotic search algorithm was developed for nonlinear constrained optimization problems [Liu Yi, 2016]. PSO and chaotic sequence methods are integrated in order to arrange a chaotic searching behavior. The logistic equation is described as in equation (13) [Hussain, et.al, 2013]:

$$
\beta k+1=\mu \beta k(1-\beta k) \quad 0 \leq \beta 1 \leq 1
$$

Where $\mu$ is the control parameter with a real value between [0,4] [16]. The inertia weight perform in (9) and (10) is typically estimated employing as describe in equation (14):

$$
\mathrm{W}=\mathrm{W}_{\max }-\left[\left(\mathrm{W}_{\max }-\mathrm{W}_{\min }\right)(\mathrm{k} \backslash \mathrm{Maxit})\right]
$$

Where $\mathrm{W}_{\text {max }}$ is the maximum value equal to 0.9 and $\mathrm{W}_{\min }$ is the minimum value of equal to 0.3 , the new inertia weight $\mathrm{W}_{\text {new }}$ is became as shown in equation (15):

$$
w_{\text {new }}=w \times \beta k+1
$$

To develop the global searching ability of simple PSO, the new update velocity will be as in equations (16 \& 17):

$$
\begin{aligned}
& V i^{x}(k+1)=W n e w V i(k)+c 1 r 1[\text { pbesti }-x i(k)]+c 2 r 2[\text { gbest }-x i(k)] \\
& V i^{y}(k+1)=W n e w V i(k)+c 1 r 1[\text { pbesti }-y i(k)]+c 2 r 2[\text { gbest }-y i(k)]
\end{aligned}
$$

Chaotic PSO algorithm can be summarized in pseudo code as shown below: 


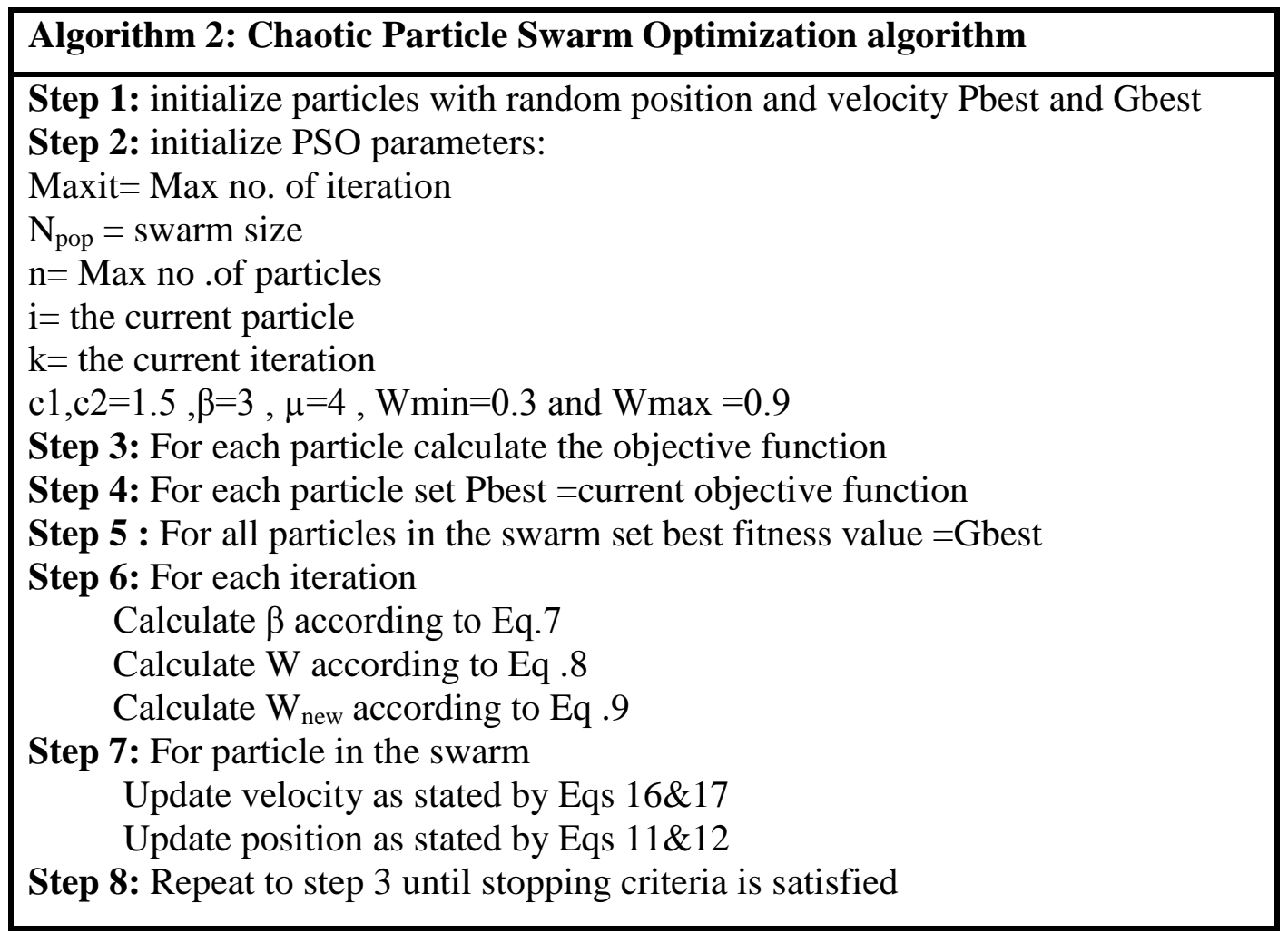

\section{WHEELED MOBILE ROBOT PATH PLANNING USING CHAOTIC PSO}

The Particle Swarm Optimization technique can be applied to the wheeled mobile robot to find a desired route while navigating in the environment.

Algorithm steps:

1- $\quad$ Make the environment as be $2 \mathrm{D}$ square map. The size of the environment is $10 \times 10$ in $(\mathrm{m} \times \mathrm{m})$. The left lowest place of the environment is the beginning point for the path while the right highest place of the environment is the end point for a path. The shape of an obstacle is circles and the size of an obstacle is variable.

2- $\quad$ The wheeled mobile robot is not a point, the dimension of the robot is added to the dimension of an obstacle to assuring the safety of robot while trying in the environment.

3- PSO technique use a swarm of particles (agents) whose locations act the possible solutions for the intended issue, with velocities are randomly initialize in the map .

4- $\quad$ The agents are evaluated using function to see how they are relative to the optimal route and the stability of the PSO algorithm. The shortest distance is the objective function which makes the mobile robot travel in the static environment with shortest time and distance as shown in equation (18):

$$
M L=\sum_{i=1}^{n p-1} \sqrt{(X(i+1)-X(i))^{2}+(Y(i+1)-Y(i))^{2}}
$$

5- The particle fitness is match with its previous best fitness Pbest for every iteration to determine the next possible coordinate position for robot in the search environment. The next possible velocity and position of each robot are determine according to (16) and (17)

6- If the next position comes into the impact of obstacle then obstacle is avoided by violation as in equations $(19,20 \& 21)$. 
Violation=violation + mean $(\mathrm{V})$

$\mathrm{V}=\max (1.2-\mathrm{D} / \mathrm{R}, 0)$

$$
\mathrm{D}=\sqrt{(x-x o b s(k))^{2}+(y-y o b s(k))^{2}}
$$

Where D is Euclidian distance between obstacle and robot, $\mathrm{R}$ is the size of obstacles, xobs and yobs are obstacles coordination.

7 - If obstacle is not present robot has to move to the next position.

8- Calculate Linear and angular velocities according to (6) and (7).

\section{SIMULATION RESULTS}

In all simulation fields , the swarm size $\mathrm{N}_{\text {pop }}=20$, Number of Way Points $\mathrm{M}=3$, Maximum number of iteration $=100$, personal factor $\mathrm{c}_{1}=2$, social factor $\mathrm{c}_{2}=2$, inertia weight damping ratio wdamp $=0.98$, distance between two wheels $\mathrm{L}=0.36 \mathrm{~m}$, radius of right and left wheel $\mathrm{r}=0.075 \mathrm{~m}$ , sampling time $T s=0.1 \mathrm{~s}, \beta=3, \mu=4$. Simulation is carried out in MATLAB R2014 environment. The MATLAB codes are run on a computer system with $8 \mathrm{G}$ RAM and core i7 CPU.

\section{Case Study 1: Path planning without obstacles}

In this case study, there is no obstacles are located in the work space, its show the shortest path is (11.3137 ). The Fig.2 and Fig.3 shows the results of PSO algorithm.

\section{Case Study 2: Path planning with eight obstacles}

In case study 2, eight obstacles with variable size are located in the static environment. All obstacles positions are recorded in Table 1 .

\section{Case Study 2.1: Standard PSO}

The optimal path with shortest distance using standard PSO is equal to (11.4709) with in iteration (47). The Fig. 4 and Fig.5 shows the converge results of Standard PSO algorithm. Finally, the reference linear velocity and the reference angular velocity of platform based on standard PSO for the optimal path are shown in Fig. 6 while the linear and angular velocities of right and left wheel are shown in Fig.7 and Fig. 8, respectively.

\section{Case Study 2.2: Chaotic PSO}

The best path with shortest distance using Chaotic PSO algorithm is equal to (11.468) in iteration (20) .The Fig. 9 and Fig. 10 shows the results of Chaotic PSO algorithm. Finally, the reference linear velocity and the reference angular velocity of platform based on standard PSO for the optimal path are shown in Fig. 11 while the linear and angular velocities of right and left wheel are shown in Fig. 12 and Fig. 13, respectively.

In addition, table 2 summarized a comparison results between two optimization algorithms. It is clearly to say that Chaotic PSO get best path length than standard version of PSO with less number of iterations.

\section{CONCLUSION}

Path planning is an important problem in mobile robot navigation. In this work a comparative study between two optimization techniques is presented to find the best route for wheeled mobile robot on a static environment. First PSO had been applied to find the best path for mobile robot, then chaotic PSO applied to enhance the results of path planning algorithm. The simulation results have shown that the path found by Chaotic PSO is shorter than the path of PSO with less number of iteration. 
Table 1: Definition of obstacles.

\begin{tabular}{|c|c|c|}
\hline Obstacle & Radius (m) & Center (xob,yob) \\
\hline 1 & 0.32 & $(1,8)$ \\
\hline 2 & 0.62 & $(1.5,4.5)$ \\
\hline 3 & 0.32 & $(3,7)$ \\
\hline 4 & 0.32 & $(4,3)$ \\
\hline 5 & 0.82 & $(9,4)$ \\
\hline 6 & 0.32 & $(8,8)$ \\
\hline 7 & 0.52 & $(6,6)$ \\
\hline 8 & 0.62 & $(9,1)$ \\
\hline
\end{tabular}

Table 2: The results of two algorithms.

\begin{tabular}{|c|c|c|}
\hline Performance & $\begin{array}{c}\text { The results of the } \\
\text { Standard PSO }\end{array}$ & $\begin{array}{c}\text { The results of the Chaotic } \\
\text { PSO }\end{array}$ \\
\hline $\begin{array}{c}\text { The best length path in } \\
(\mathrm{m})\end{array}$ & 11.4709 & 11.468 \\
\hline $\begin{array}{c}\text { The iteration of Best } \\
\text { Path }\end{array}$ & 47 & 20 \\
\hline Execution Time $(\mathrm{sec})$ & 28.4887 & 25.2379 \\
\hline
\end{tabular}

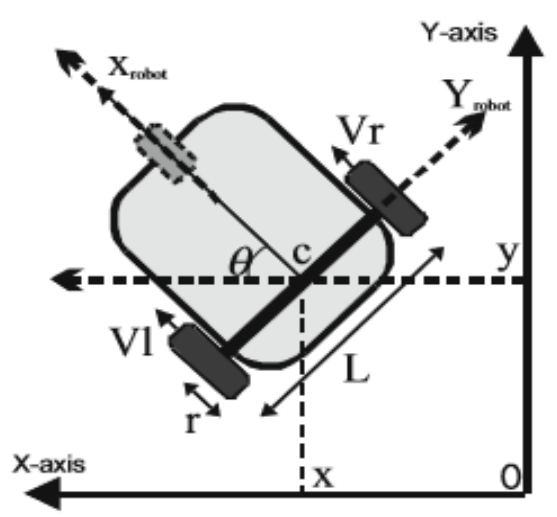

Fig. 1: Mobile Robot Model [1]. 


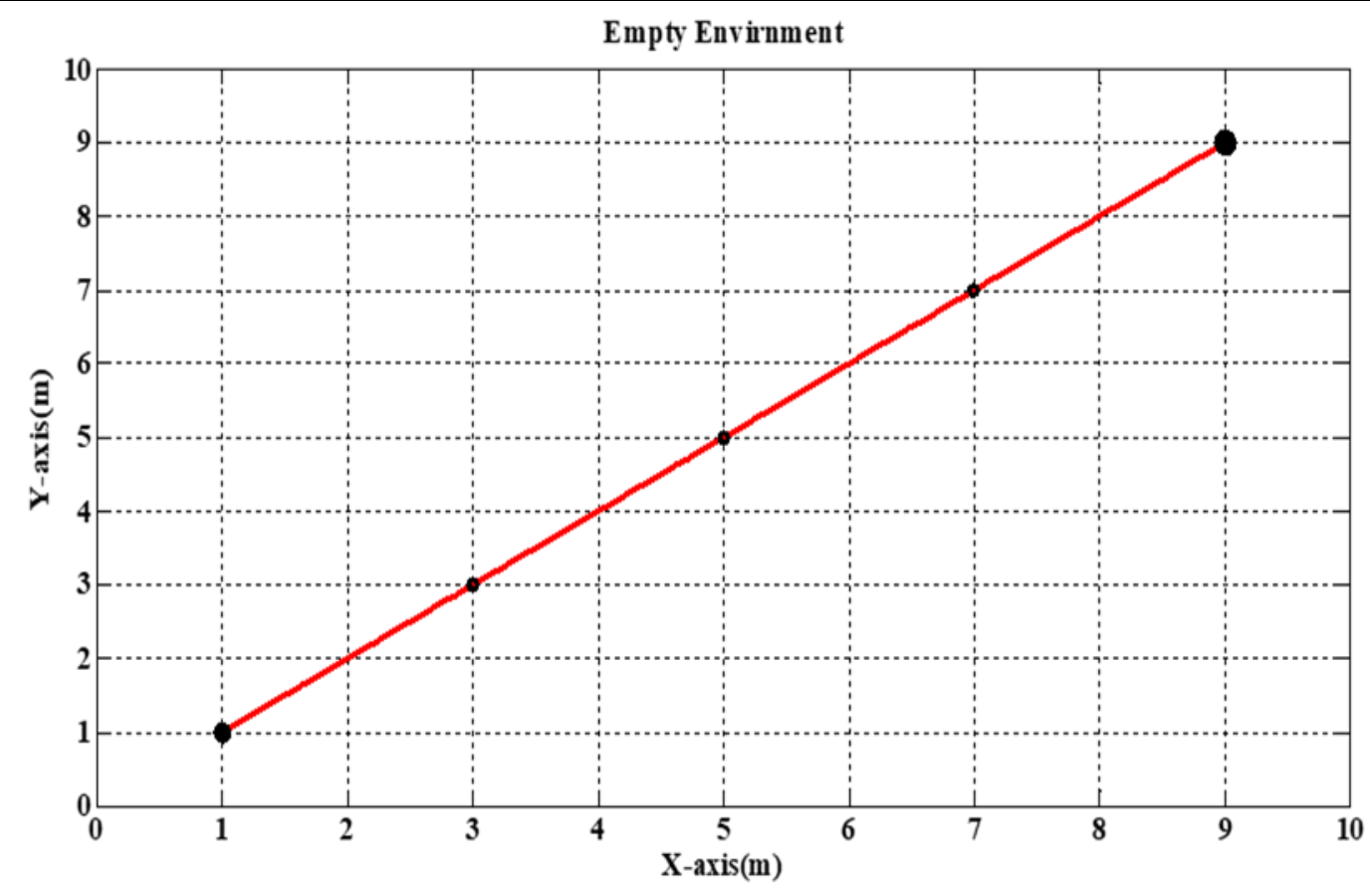

Fig. 2: Path Planning with Empty Map.

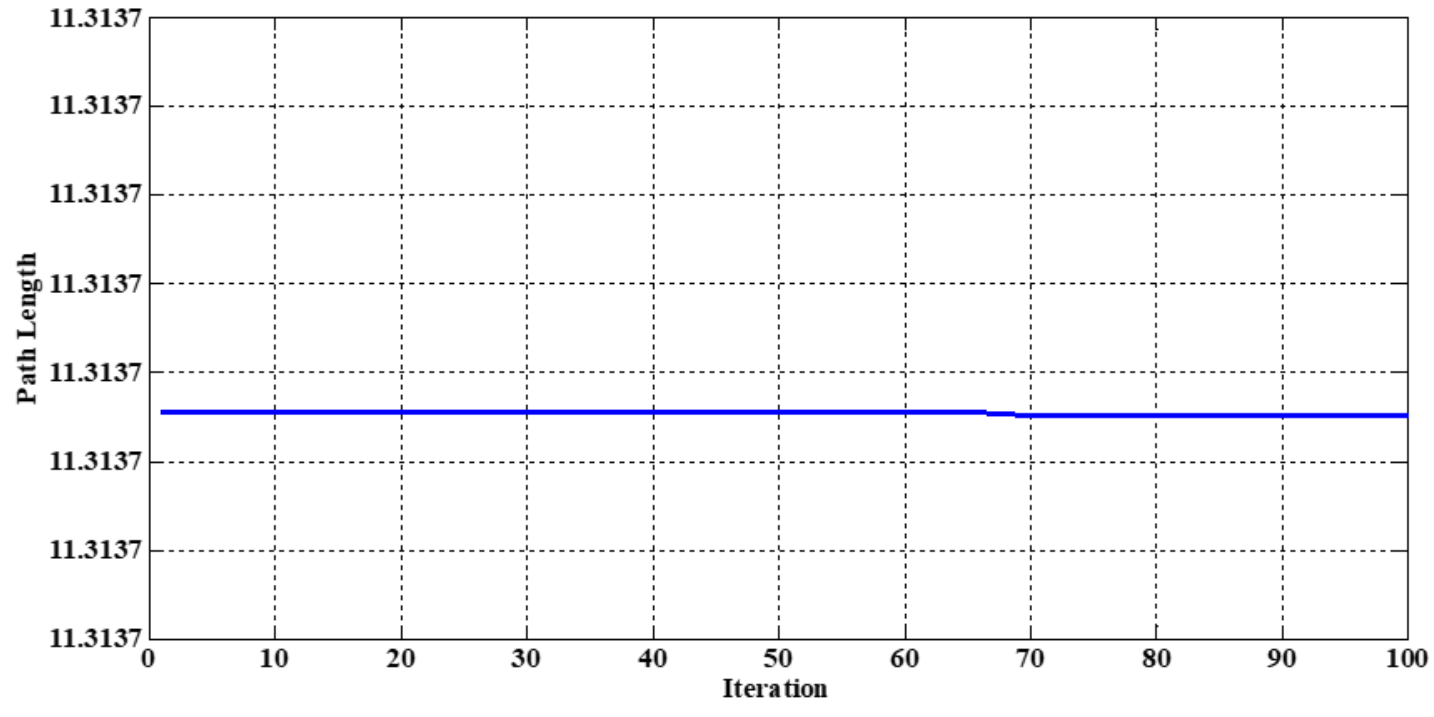

Fig. 3: The convergence Curve for empty map. 


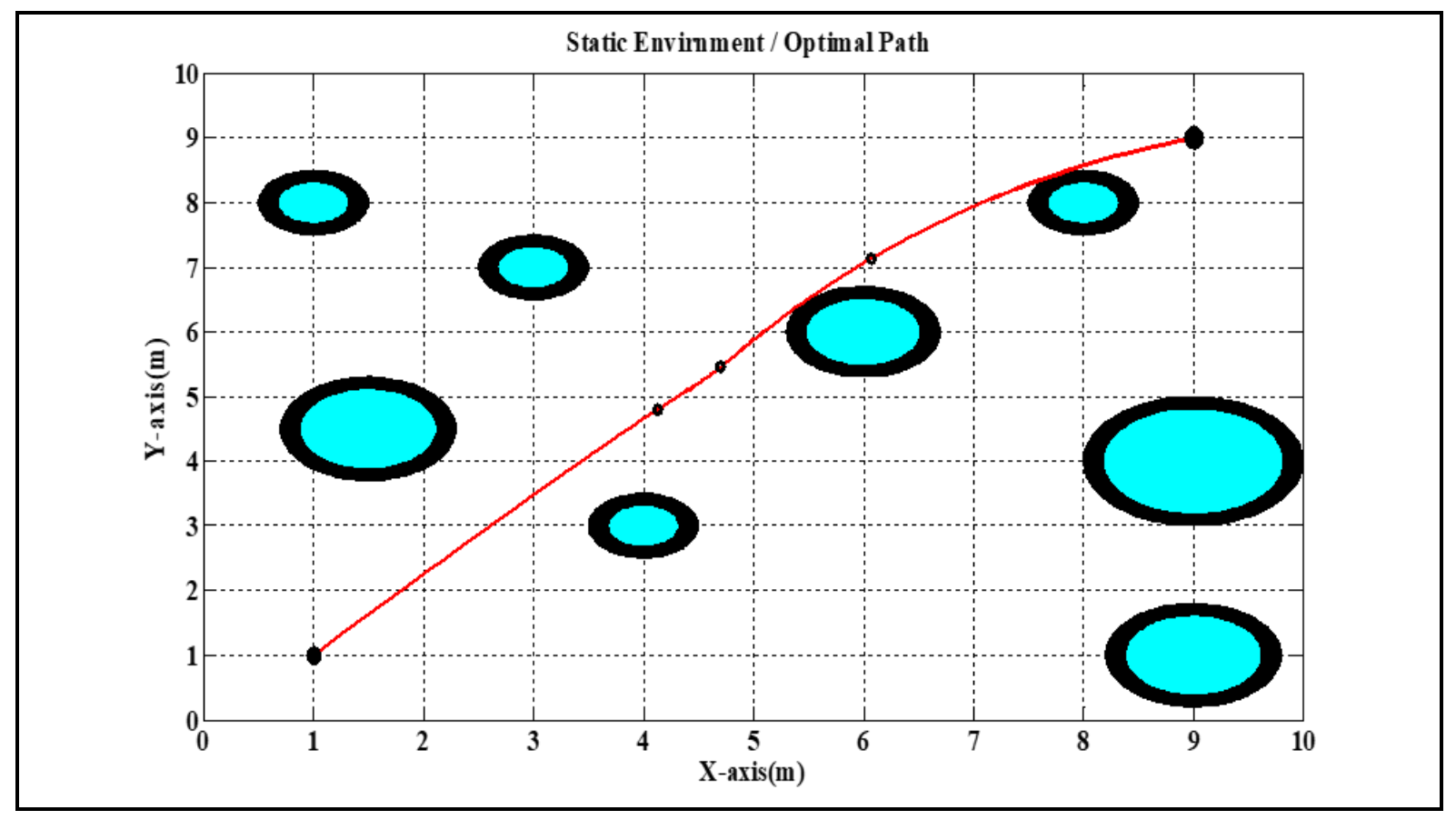

Fig. 4: Best path found by Standard PSO.

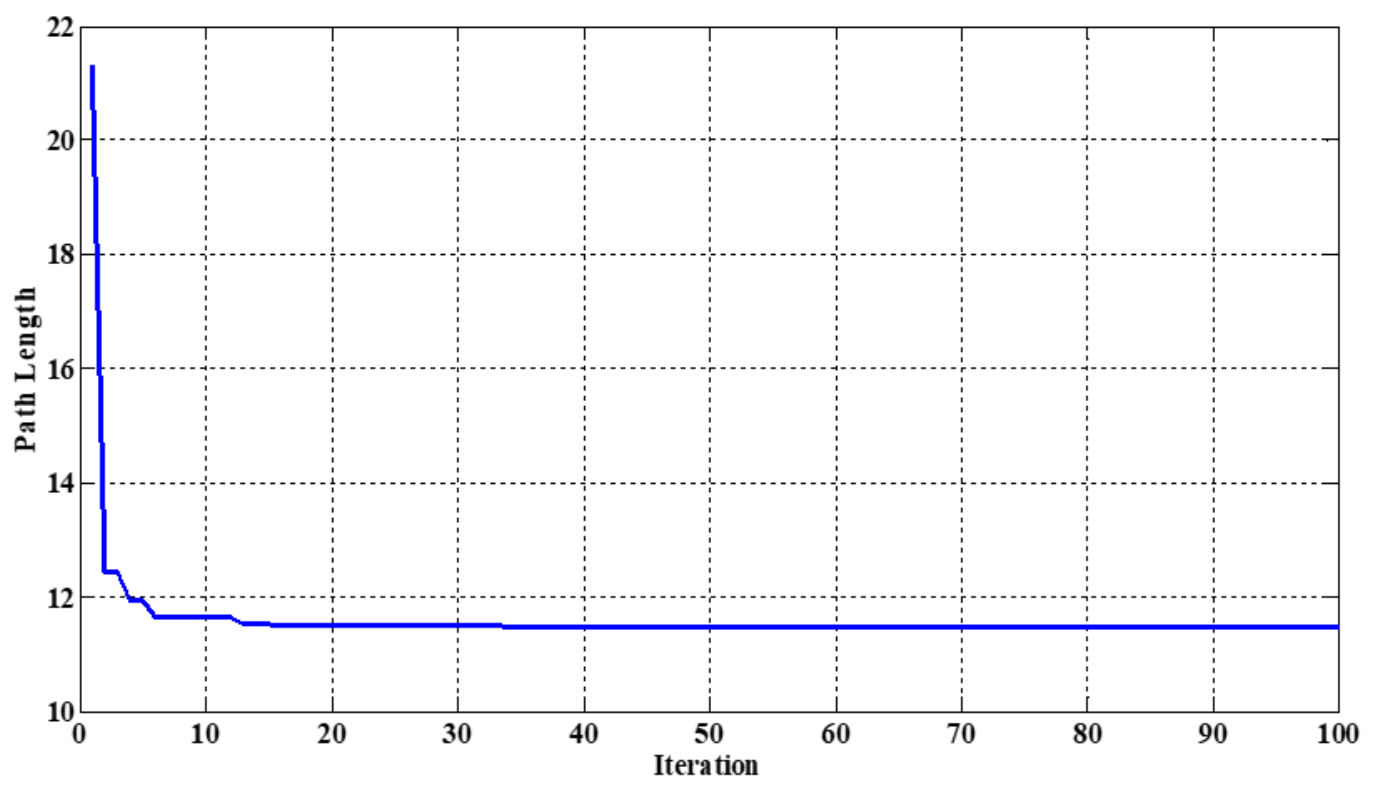

Fig. 5: The convergence Curve for Standard PSO algorithm. 


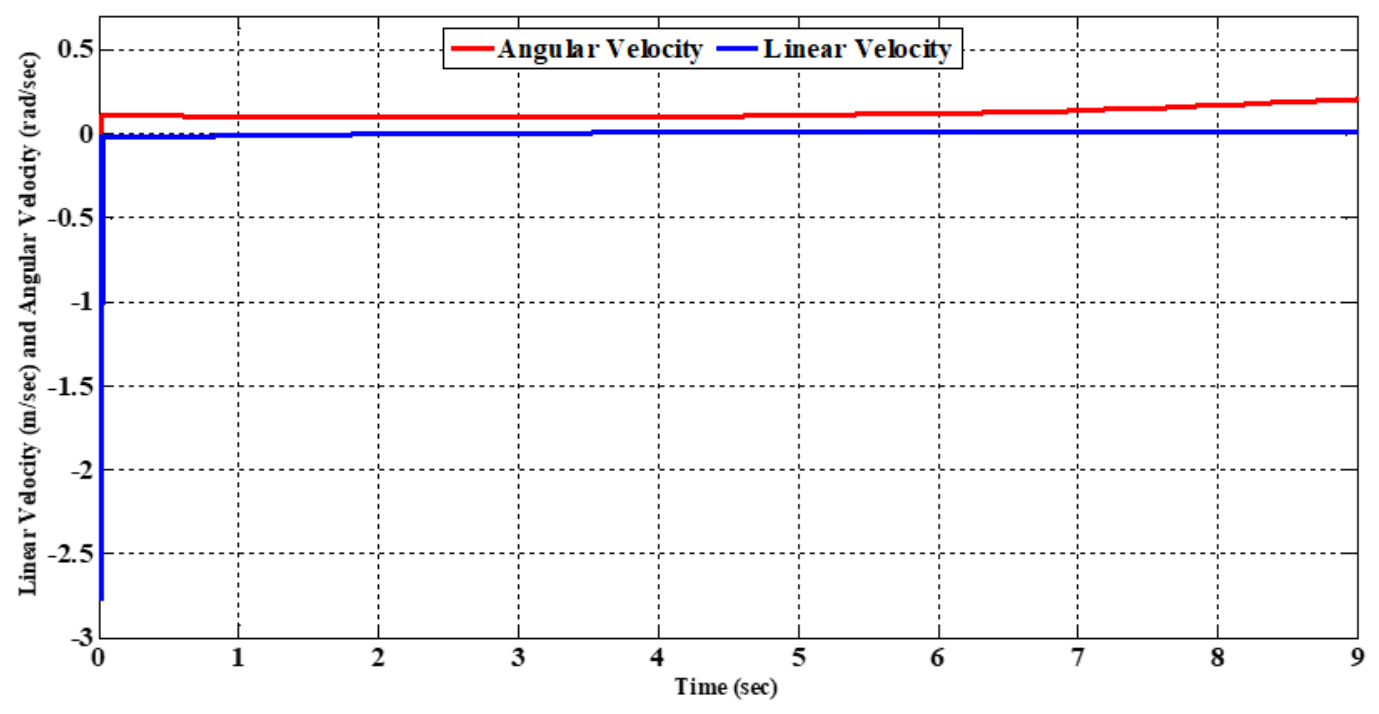

Fig. 6: Linear and Angular Velocity of platform based on PSO.

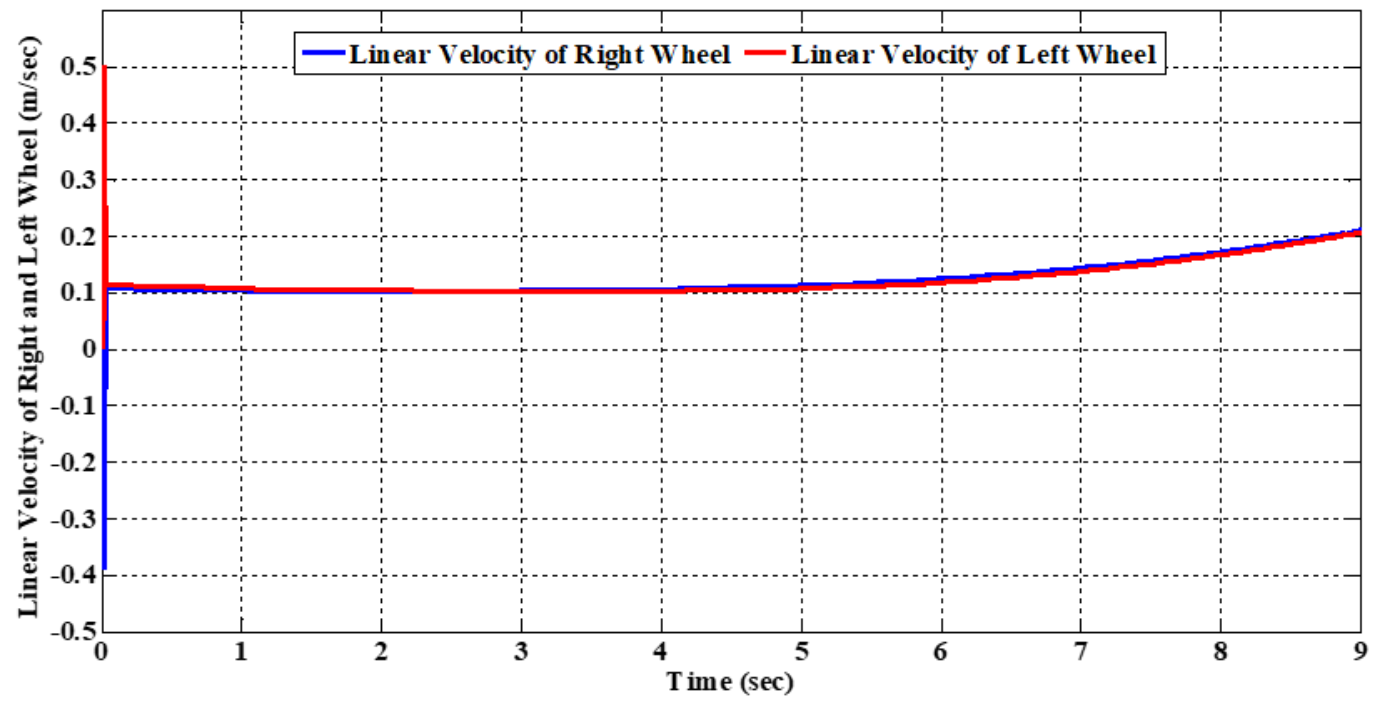

Fig. 7: Linear Velocity of right and left wheel based on PSO. 


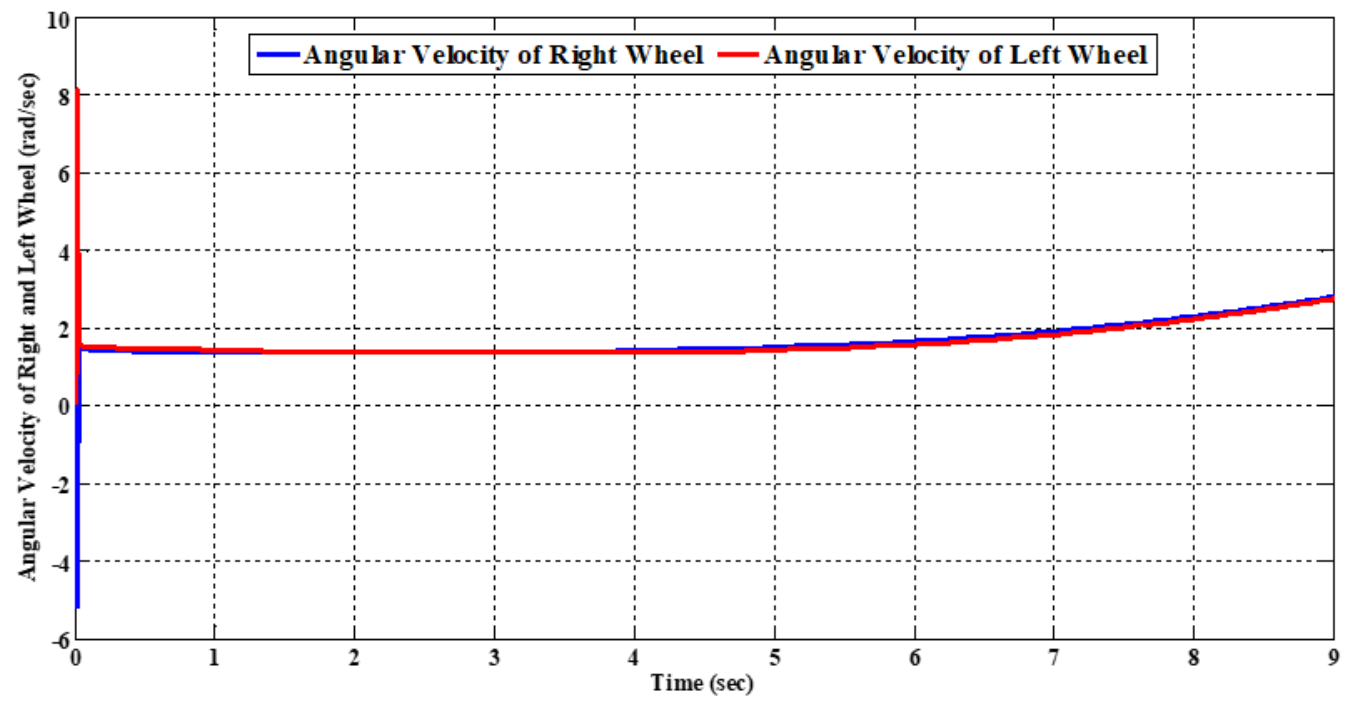

Fig. 8: Angular Velocity of right and left Wheel based on PSO.

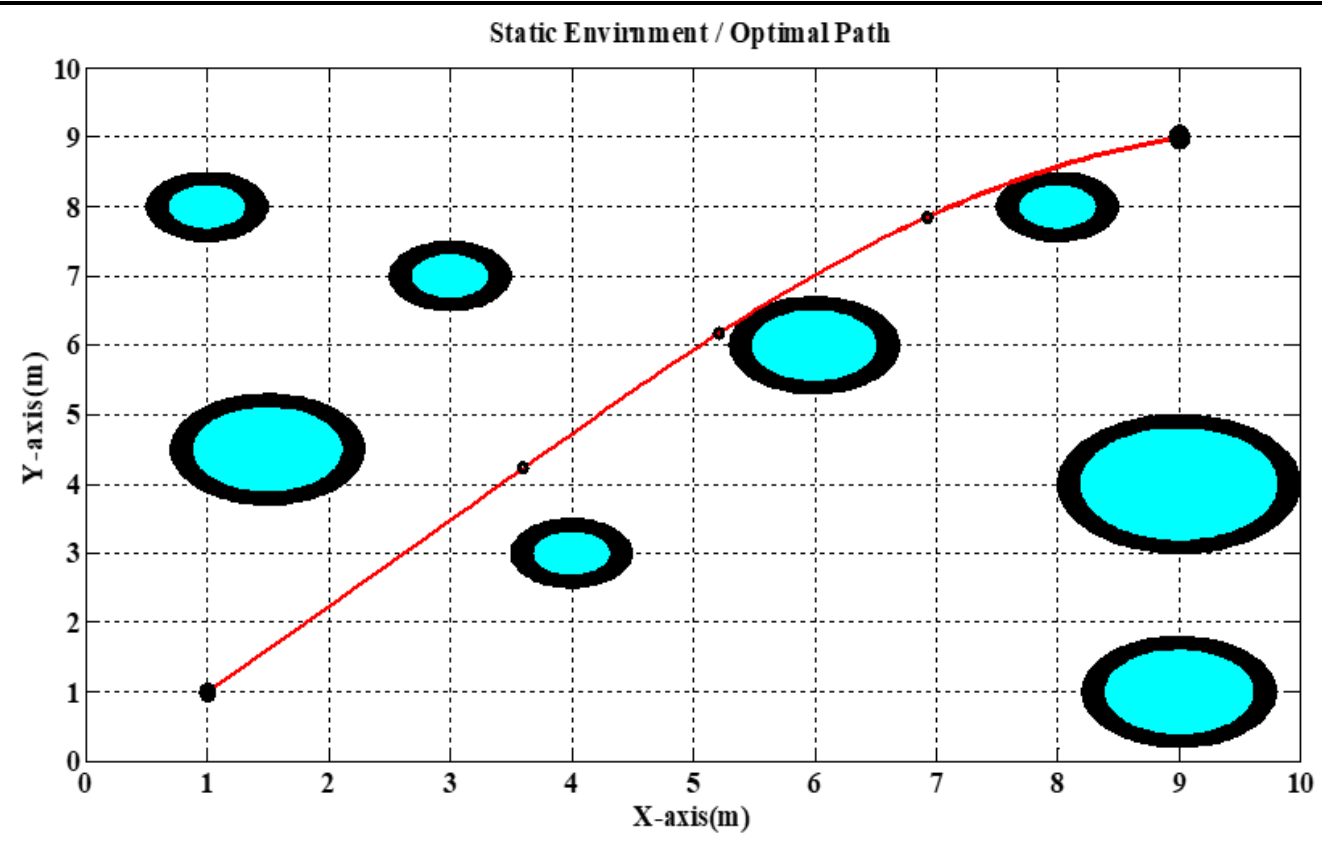

Fig. 9: Best Path found by Chaotic PSO. 


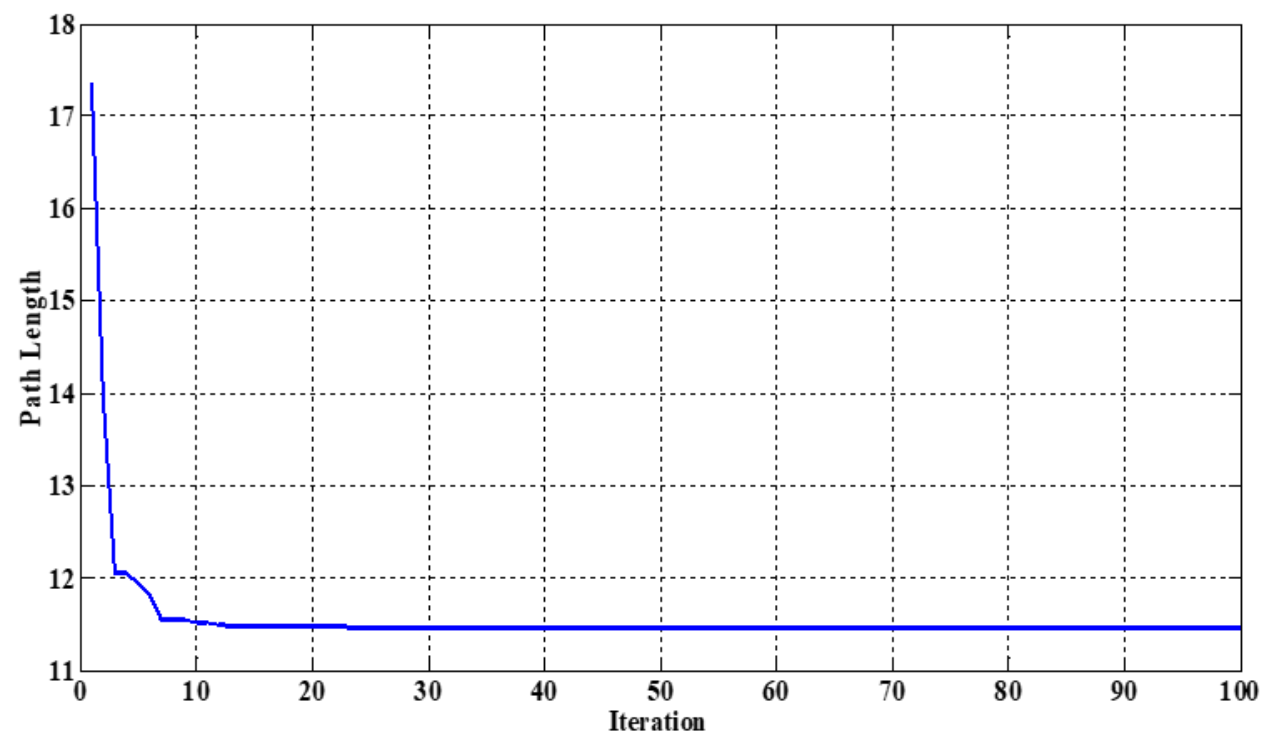

Fig. 10: The Convergence Curve for Chaotic PSO.

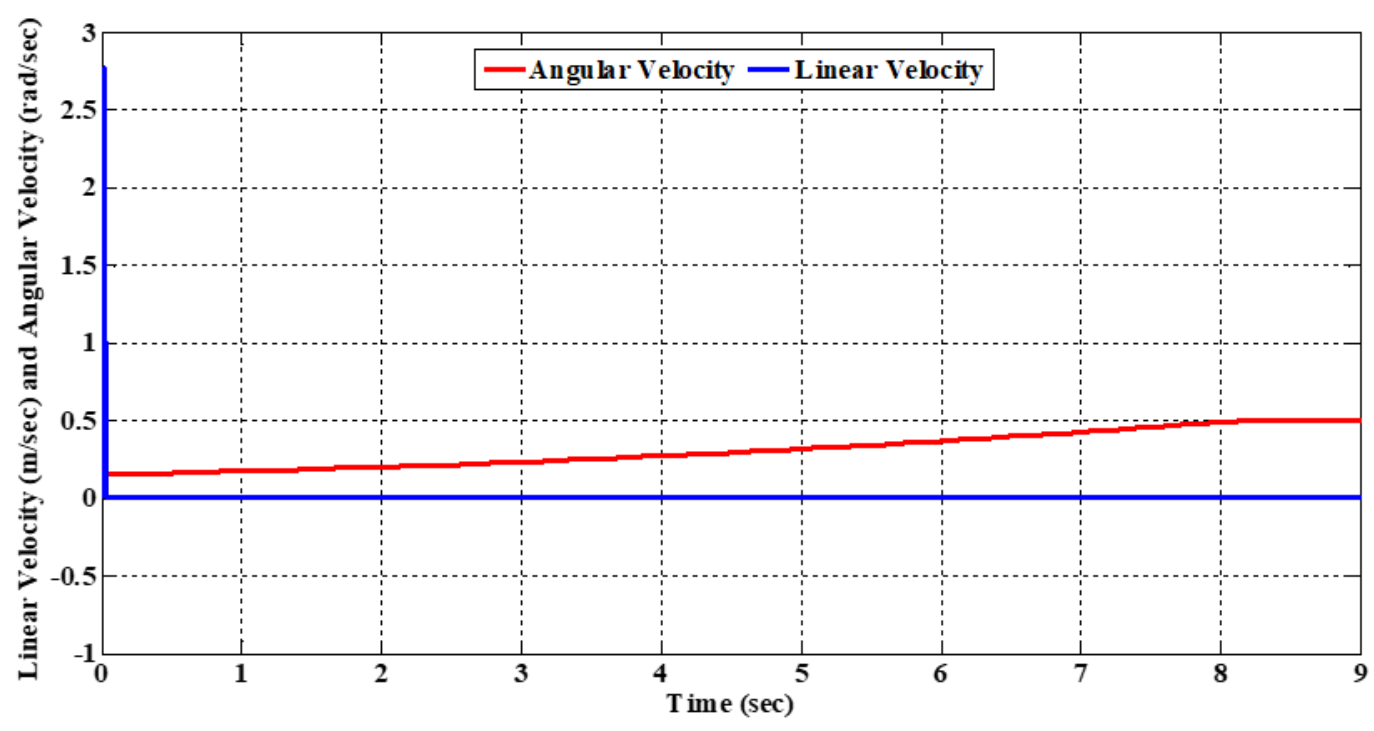

Fig. 11: Linear Velocity and angular Velocity of platform based CPSO. 


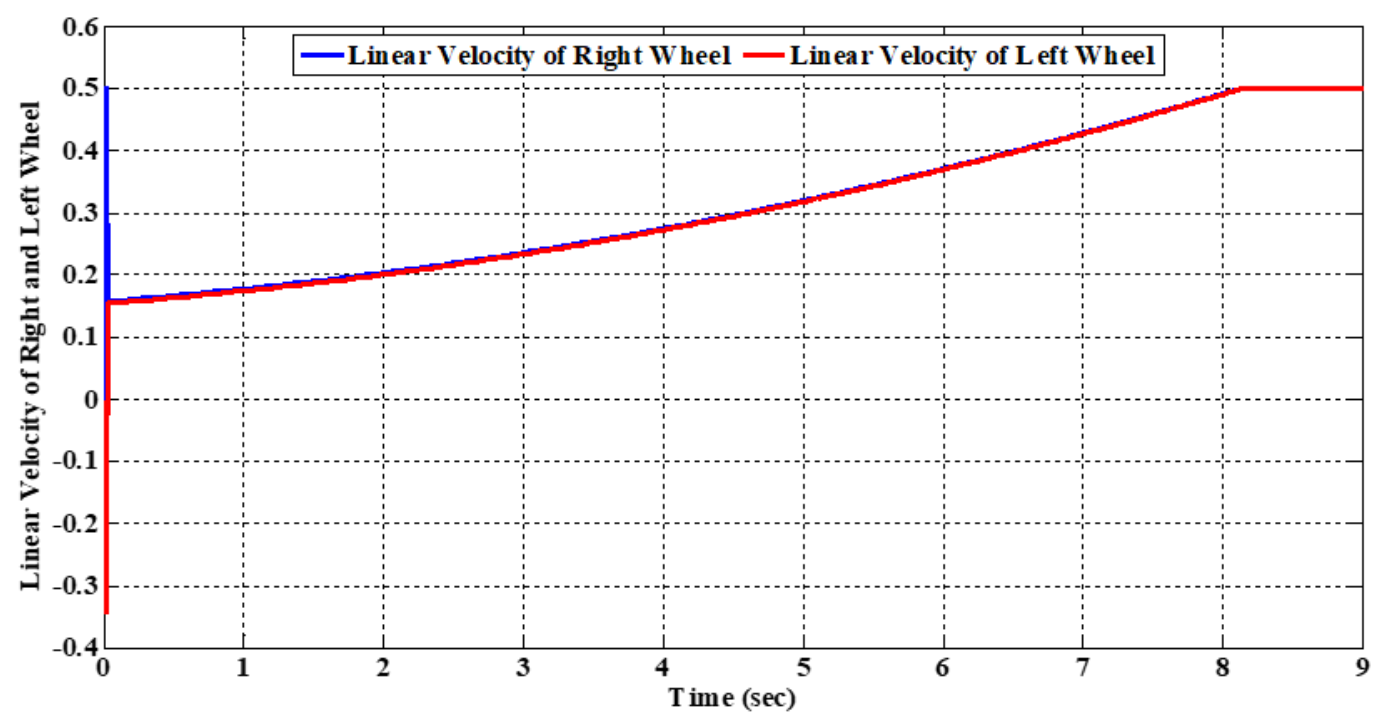

Fig. 12: Linear Velocity of right wheel and left wheel based on CPSO.

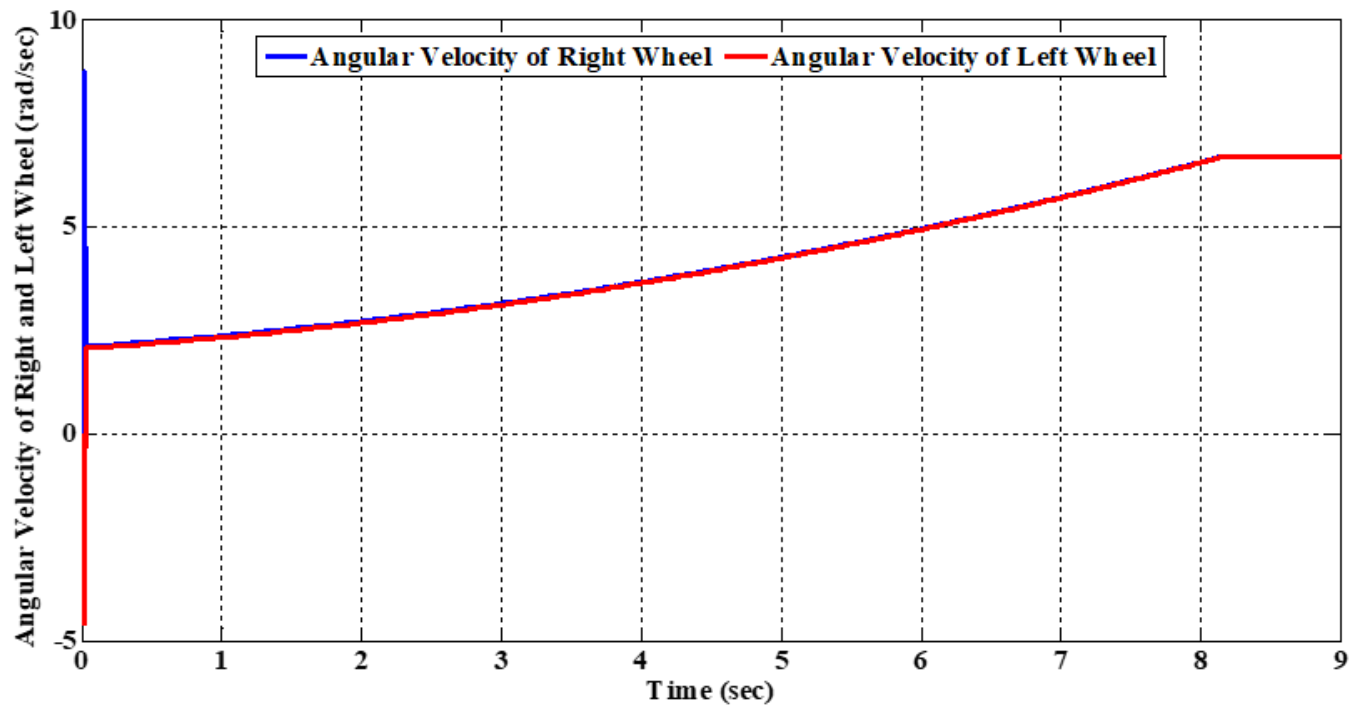

Fig. 13: Angular Velocity of right wheel and left wheel based on CPSO.

\section{REFERENCES}

A. S. Al-Araji, 2014 "Development of Kinematic Path -Tracking Controller Design for Real Mobile Robot via Back - Stepping Slic Genetic Robust Algorithm Technique", Arabian Journal for Science and Engineering, Vol. 39, No. 12, PP.(8825-8835).

E. Cholodowicz and D.Figurowski, 2017 "Mobile Robot Path Planning with Obstacle Avoidance using Particle Swarm Optimization“, Pomiary Automatyka Robotyka ,R.21,Nr. 3,PP. (59-68).

N. H. Abbas and F. M. Ali, 2016 " Path Planning of an Autonomous Mobile Robot using Enhanced Bacterial Foraging Optimization Algorithm “, Al-Khwarizmi Engineering Journal, Vol.12, No.4, PP.(26-35). 
S. A. Mnubi, 2016" Motion Planning and Trajectory for Wheeled Mobile Robot, “, International Journal of Science and Research, Vol. 5 Issue 1, PP. (1064-1068).

S. Jalel, P.Marthon and A. Hamouda, 2015" NURBS Based Multi-objective Path Planning “, Springer International Publishing Switzerland, PP. (190-199).

N. H. Abbas and F. M. Ali, 2014 "Path planning of an Autonomous Mobile Robot using Directed Artificial Bee Colony Algorithm", International Journal of Computer Application, Vol. 96, No. 11, PP.(11-16)

T. A. Jaleel and A. K. Abdul Hassan, 2016 " Collision Avoidance Using Cat Swarm Algorithm for Multi Mobile Robot Path Planning in Dynamic Environment" , Iraqi Journal of Science, Vol. 57, No. 3C, PP.(2348-2359)

A. A. Ahmed, T. Y. Abdalla and A. A. Abed, 2015 " Path Planning of Mobile Robot Using Fuzzy-Potential Field Method”, Iraq J. Electrical and Electronic Engineering, Vol.11, No.1, PP.

A. S. Al-Araji, 2014 "Design of On-Line Nonlinear Kinematic Trajectory Tracking Controller for Mobile Robot based on Optimal Back-Stepping Technique”, Iraqi Journal of Computers, Communication and Control \& Systems Engineering, Vol. 14, No. 2, PP. (25-36)

A. S. Al-Araji, M. F. Abbod, H. S. Al-Raweshidy, 2011 "Neural autopilot predictive controller for nonholonomic wheeled mobile robot based on a pre-assigned posture identifier in the presence of disturbances", The 2nd International Conference on Control, Instrumentation and Automation (ICCIA), PP.(326-331).

A. S. Al-Araji, M. F. Abbod, H. S. Al-Raweshidy, 2011 "Design of an adaptive nonlinear PID controller for nonholonomic mobile robot based on posture identifier", The IEEE International on Control System, Computing and Engineering (ICCSCE), PP. (337-342).

J. A. Abdulsahib, 2016 "Improvement of Multi-Objective Path Planning for Multi-Robot Systems Using Adaptive PSO and ABC Algorithms", MSc Thesis, University of Baghdad.

N. H. Abbas, J. A. Abdulsaheb, 2016 " An Adaptive Multi- Objective Particle Swarm Optimization Algorithm for Multi - Robot Path Planning “, Journal of Engineering, No. 7, Vol. 22, PP.(164-180).

Z. Assarzadeh and A. R. Naghsh - Nilchi, 2015"Chaotic Particle Swarm Optimization with mutation for classification”, Journal of Medical Signals and Sensors, Vol. 5, PP. (12-20).

Liu Yi, 2016 "Study on an improved PSO Algorithm and its Application for Solving Function Problem", International Journal of Smart Home, Vol. 10, No. 3, PP.(51-62).

A. N. Hussain , F. Melk , M. A. Rashid, L. Moamed, and N. A. Mohd Affendi, 2013 " Optimal Coordinated Design of Multiple Damping Controllers Based on PSS and UPFC Device to Improve Dynamic Stability in the Power System", Hindawi Publishing Corporation Mathematical Problem in Engineering, PP.(1-15). 